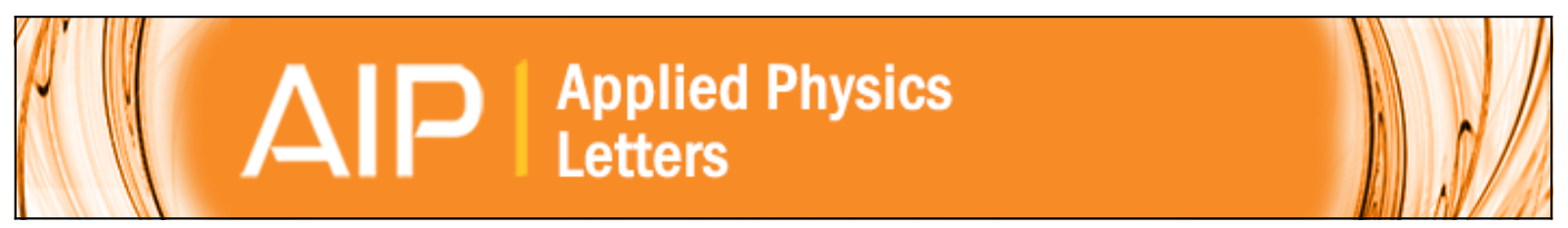

\title{
Electric field engineering using quantum-size-effect-tuned heterojunctions
}

V. Adinolfi, Z. Ning, J. Xu, S. Masala, D. Zhitomirsky, S. M. Thon, and E. H. Sargent

Citation: Applied Physics Letters 103, 011106 (2013); doi: 10.1063/1.4813074

View online: http://dx.doi.org/10.1063/1.4813074

View Table of Contents: http://scitation.aip.org/content/aip/journal/apl/103/1?ver=pdfcov

Published by the AIP Publishing

\section{Articles you may be interested in}

Photocurrent extraction efficiency in colloidal quantum dot photovoltaics

Appl. Phys. Lett. 103, 211101 (2013); 10.1063/1.4831982

Systematic optimization of quantum junction colloidal quantum dot solar cells

Appl. Phys. Lett. 101, 151112 (2012); 10.1063/1.4757866

Effect of ZnS coating on the photovoltaic properties of CdSe quantum dot-sensitized solar cells J. Appl. Phys. 103, 084304 (2008); 10.1063/1.2903059

Efficient solution-processed infrared photovoltaic cells: Planarized all-inorganic bulk heterojunction devices via inter-quantum-dot bridging during growth from solution

Appl. Phys. Lett. 90, 183113 (2007); 10.1063/1.2735674

Quantum box size effect on vertical self-alignment studied using cross-sectional scanning tunneling microscopy Appl. Phys. Lett. 74, 2608 (1999); 10.1063/1.123912

\section{AIP $\mid$ APL Photonics}

APL Photonics is pleased to announce Benjamin Eggleton as its Editor-in-Chief

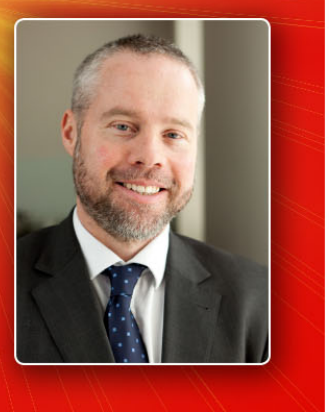




\title{
Electric field engineering using quantum-size-effect-tuned heterojunctions
}

\author{
V. Adinolfi, ${ }^{1}$ Z. Ning, ${ }^{1}$ J. Xu, ${ }^{1}$ S. Masala,${ }^{2}$ D. Zhitomirsky, ${ }^{1}$ S. M. Thon, ${ }^{1}$ and E. H. Sargent ${ }^{1}$ a) \\ ${ }^{1}$ Department of Electrical and Computer Engineering, University of Toronto, 10 King's College Road, \\ Toronto, Ontario M5S 3G4, Canada \\ ${ }^{2}$ Division of Physical Sciences and Engineering, Solar and Photovoltaics Engineering Center, \\ King Abdullah University of Science and Technology (KAUST), Thuwal 23955-6900, Saudi Arabia
}

(Received 19 December 2012; accepted 16 June 2013; published online 3 July 2013)

A quantum junction solar cell architecture was recently reported that employs colloidal quantum dots (CQDs) on each side of the p-n junction. This architecture extends the range of design opportunities for CQD photovoltaics, since the bandgap can be tuned across the light-absorbing semiconductor layer via control over CQD size, employing solution-processed, roomtemperature fabricated materials. We exploit this feature by designing and demonstrating a field-enhanced heterojunction architecture. We optimize the electric field profile within the solar cell through bandgap engineering, thereby improving carrier collection and achieving an increased open circuit voltage, resulting in a $12 \%$ improvement in power conversion efficiency. (C) 2013 AIP Publishing LLC. [http://dx.doi.org/10.1063/1.4813074]

Colloidal quantum dot (CQD) solids are promising semiconductor materials for electronic and optoelectronic applications. A variety of devices utilizing these materials have been realized so far, including light-emitting devices, ${ }^{1}$ photodetectors, ${ }^{2,3}$ field effect transistors, ${ }^{4,5}$ and photovoltaics. ${ }^{6-8}$ CQD films offer several distinct advantages as an optoelectronic material, including inexpensive and facile processing based on spin-casting, dip-coating, and spray-coating, a high optical absorption coefficient, ${ }^{9}$ and the ability to tune the bandgap via the quantum size effect.

In photovoltaic applications, p-type CQD films are typically combined with an n-type wide bandgap semiconductor $^{10}$ to create a $\mathrm{p}-\mathrm{n}$ heterojunction. This architecture has resulted in improved power conversion efficiencies, but is unable to take advantage of the bandgap tuning afforded by the CQD films because of the requirements imposed by band alignment with the wide-bandgap semiconductor. ${ }^{11}$ Recently, an all-CQD rectifying p-n homojunction solar cell, termed the Quantum Junction (QJ), was reported, ${ }^{12}$ consisting of both p-type and n-type CQD solids to form the $\mathrm{p}-\mathrm{n}$ junction. This architecture extends the range of design opportunities for CQD photovoltaics, since the bandgap can be tuned, in a spatially varying fashion, across the lightabsorbing semiconductor layer by changing the CQD size. Attractively, this active region consists of solutionprocessed, room-temperature fabricated materials.

We sought to improve further upon the QJ concept by investigating architectures that optimize the electric field profile for photovoltaic performance within the active material. Currently, power conversion efficiencies in CQD solar cells are limited by low currents due to short diffusion lengths ${ }^{13}$ and by low voltages due to trap-state assisted recombination. ${ }^{14}$ Our architecture, termed the field-enhanced QJ, employs a large bandgap layer at the far side of the n-type film. This strategy is expected to improve carrier collection and the open circuit voltage $\left(\mathrm{V}_{\mathrm{OC}}\right)$ by improving

\footnotetext{
${ }^{\text {a) }}$ Author to whom correspondence should be addressed. Electronic mail: ted.sargent@utoronto.ca.
}

the electric field profile within the active layer and increasing quasi-Fermi level separation, respectively.

The QJ and field-enhanced QJ device architectures are illustrated in Figures 1(a) and 1(b). The QJ device consist of a $\mathrm{PbS}$ CQD rectifying p-n junction between a thin, highly doped $\mathrm{p}+$ type CQD layer and a lightly doped n-type CQD layer where a wide depletion region is established, promoting efficient collection of the electric charge. ${ }^{12}$ Solar radiation illuminates the $\mathrm{p}+$ side of the junction. Indium tin oxide (ITO) and aluminum-doped zinc oxide (AZO) are used as ohmic contacts to the $\mathrm{p}$ and $\mathrm{n}$ layers, respectively. ${ }^{12}$

In the field-enhanced QJ device, a thin layer of n-type quantum dots with slightly smaller nanoparticle diameter (higher bandgap) than those used in the p-n junction layers is introduced on top of the n-type layer. Figures 1(a) and 1(b) show simulated band diagrams at equilibrium for the QJ and the field-enhanced QJ architectures. For PbS quantum dots with excitonic energies near $1.3 \mathrm{eV}$, the tuning of the bandgap occurs mostly in the conduction band, ${ }^{15}$ resulting in an increase of the conduction band energy at the top of the ntype layer for the field-enhanced QJ device.

The field-enhanced QJ is expected to result in improved power conversion efficiency compared to the QJ by increasing both $\mathrm{V}_{\mathrm{OC}}$ and $\mathrm{J}_{\mathrm{SC}}$. The doping densities of the small (n) and large $(\mathrm{N})$ bandgap n-type layers are kept constant, as confirmed from analysis of capacitance-voltage measurements (see supplementary material). ${ }^{16}$ Assuming that the effective density of states in the conduction and in the valence band $\left(\mathrm{N}_{\mathrm{C}}\right.$ and $\left.\mathrm{N}_{\mathrm{V}}\right)$ does not experience large variations for different bandgap PbS CQD solids, the Fermi levels within the isolated $\mathrm{n}$ and $\mathrm{N}$ layers are equally distant from the respective conduction band edges. ${ }^{17}$ This leads to increased separation between the Fermi levels of the isolated $\mathrm{p}+$ and $\mathrm{N}$ layers compared to the Fermi level separation between the isolated $\mathrm{p}+$ and $\mathrm{n}$ material. This results in an increase of the built-in voltage in the field-enhanced QJ, and, under illumination, a larger splitting of the quasi-Fermi energies, enhancing the $\mathrm{V}_{\mathrm{OC}}$ as shown in Figures $1(\mathrm{c})-1(\mathrm{e}){ }^{18}$ Figure 1(e) shows the electric field profile, which does not 
Quantum Junction

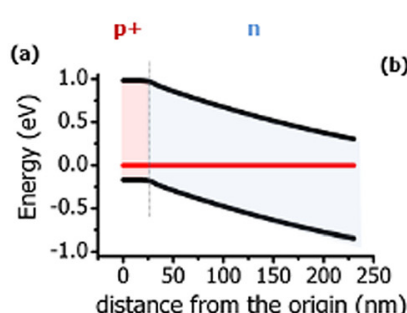

(b)
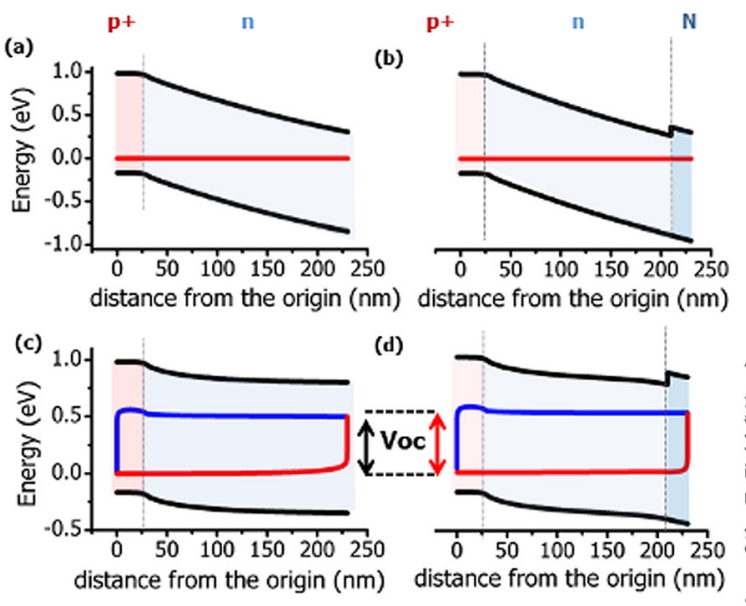

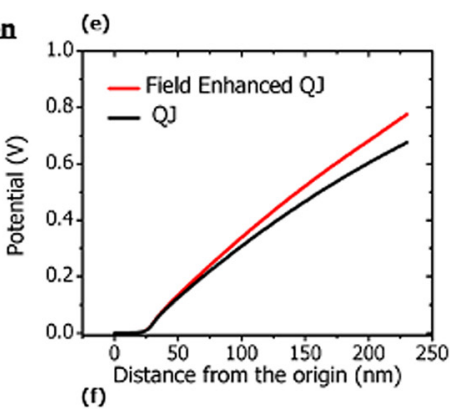

(f)

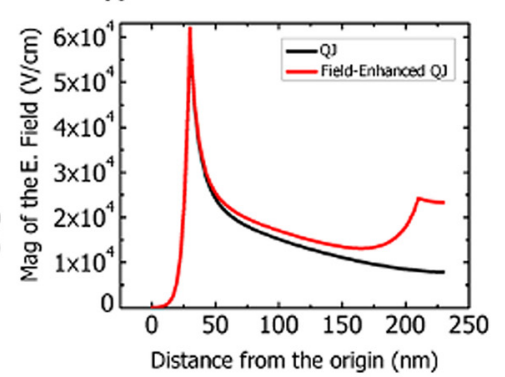

FIG. 1. (a) and (b) Simulated equilibrium band diagrams of the QJ and the field-enhanced QJ structures, respectively. (c) and (d) Simulated band diagrams at open circuit conditions for the same structures as in (a) and (b), respectively, under $1000 \mathrm{~W} / \mathrm{m}$ solar illumination. The field-enhanced QJ exhibits a higher open circuit voltage due to the increased separation between the Fermi levels of the p-type and N-type layers and (e) a higher built-in voltage at the equilibrium. (f) Simulated electric field inside the solar cell active layers at the maximum power point. The fieldenhanced QJ architecture exhibits a higher electric field magnitude. The electric field vector is directed towards the p-layer, driving minority holes towards their respective contact during photovoltaic operation. vanish throughout the $\mathrm{n}$ side of the junction for both devices since the material is fully depleted. We also note the formation of a spike in the electric field profile, as shown in Figure $1(\mathrm{f})$, as a result of the $\mathrm{n}-\mathrm{N}$ junction; this reflects a formation of a depletion region due to the differences in Fermi levels. Integrating over this electric field yields a higher built-in voltage. In this way, electric field engineering in the fieldenhanced QJ architecture can result in an increase in $\mathrm{V}_{\mathrm{OC}}$, as depicted in Figure 1(d). In effect, this architecture presents a bandgap-tuning-based alternative to doping-density-based control of the Fermi level, an advantage since doping control in high-photovoltaic-quality CQD solids is, at present, limited. ${ }^{19,20}$

In order to investigate the operation of the proposed architecture, we simulated the performance of fieldenhanced QJ devices for different values of the top $\mathrm{N}$ layer electronic bandgap. ${ }^{21-23}$ The overall thickness of the active layer (p-type film, n-type film, and N-type film) in all cases was $230 \mathrm{~nm}$. We used a highly doped $\mathrm{p}+$ layer model based on previously published device structures ${ }^{24}$ with a thickness of $30 \mathrm{~nm}$. The modeled top $\mathrm{N}$ layer was also kept thin at $20 \mathrm{~nm}$ in thickness in order to ensure that an optimal singlejunction bandgap material was employed for the preponderance of the active layer. ${ }^{25}$ Figure 2 shows the results of the simulations for (a) $\mathrm{V}_{\mathrm{OC}}$, (b) short circuit current density $\left(\mathrm{J}_{\mathrm{sc}}\right)$, (c) fill factor (FF), and (d) power conversion efficiency (PCE). The values corresponding to the smallest $\mathrm{N}$ layer electronic bandgap represent the QJ structure. $\mathrm{V}_{\mathrm{OC}}$ increases proportionally to the increase in the $\mathrm{N}$ layer bandgap. $\mathrm{J}_{\mathrm{sc}}$ and PCE exhibit maxima at intermediate values of the $\mathrm{N}$ layer electronic bandgap. We explain this behavior by considering two competing mechanisms that affect $\mathrm{J}_{\mathrm{SC}}$. The initial (a)

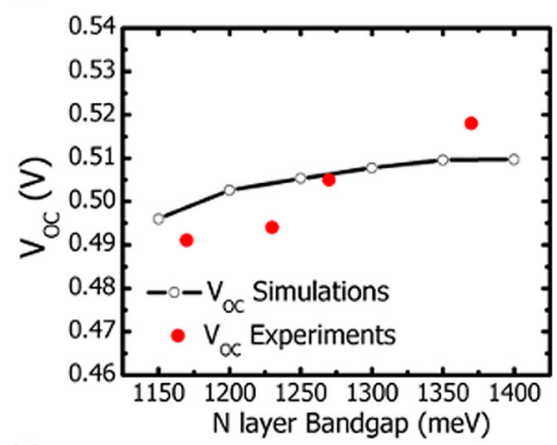

(c)

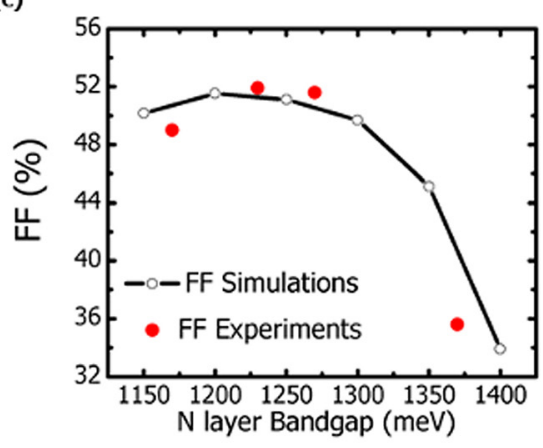

(b)

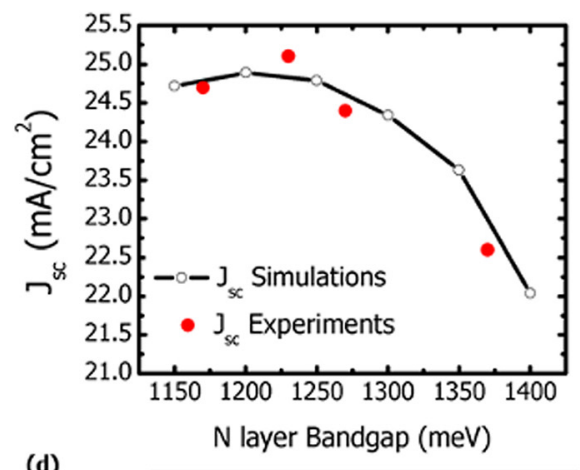

(d)

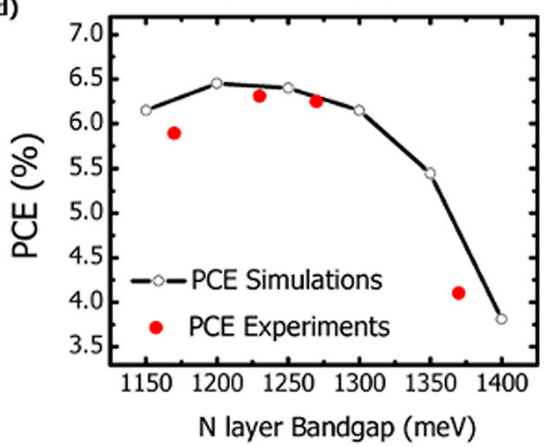

FIG. 2. Comparison between experiments and simulations for (a) open circuit voltage, (b) short circuit current, (c) fill factor, and (d) power conversion efficiency as a function of the electronic bandgap of the top $\mathrm{N}$ layer. The smallest bandgap point is equivalent to the quantum junction device. 
(a)

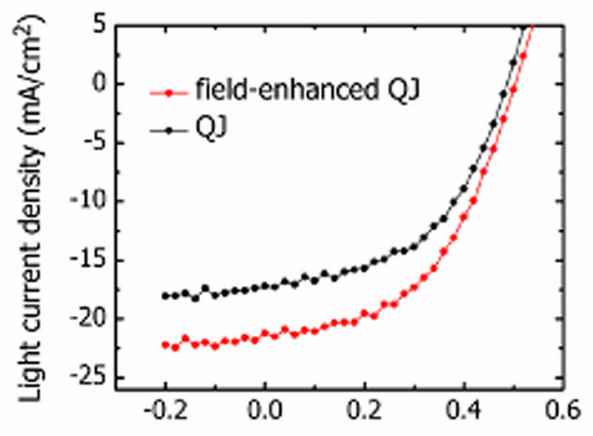

(b)

\begin{tabular}{|c|c|c|}
\hline & QJ & field-enhanced QJ \\
\hline $\mathbf{V}_{\mathbf{O C}}$ & 0.49 & 0.50 \\
\hline $\mathbf{J}_{\mathbf{s C}}$ & 24.7 & 26.4 \\
\hline $\mathbf{P C E}$ & $5.89 \%$ & $6.59 \%$ \\
\hline $\mathbf{F F}$ & $49 \%$ & $50 \%$ \\
\hline
\end{tabular}

FIG. 3. (a) Current-voltage characteristic of a typical QJ device and the best field-enhanced QJ device. (b) Comparison of device figures of merit for the QJ and optimized field-enhanced QJ structures.

increase in current as the $\mathrm{N}$ layer bandgap is increased is explained by the enhancement in electric field magnitude within the solar cell, which improves extraction of the minority charge carriers in this region. However, this increase in field amplitude is accompanied by the introduction of a step in the conduction band at the $\mathrm{n}-\mathrm{N}$ interface that constitutes a barrier for the electron flow. ${ }^{26}$ This barrier increases in height as the $\mathrm{N}$ layer electronic bandgap increases. When it reaches a critical value, a decrease in current is observed, compromising the operation of the device. Analogous considerations can be used to explain the trend in the FF. As a further element of analysis, we simulated the EQE response using illumination from the $\mathrm{n}$ side (top) for both QJ and field-enhanced QJ. The field-enhanced QJ shows an increased efficiency for short wavelengths compared to the QJ. This confirms the enhancement in photocharge collection provided by the $\mathrm{n}-\mathrm{N}$ interface. The results are presented in the supplementary material. ${ }^{16}$

Next, we sought to use the insights gained from simulation to build a series of field- enhanced QJ devices. Our device fabrication process was modified from a process previously developed for the QJ architecture. ${ }^{24}$ The p-type
TABLE I. Mean value of PCE and statistical error evaluated on 20 best QJ and field-enhanced QJ devices.

\begin{tabular}{lcc}
\hline \hline & QJ & Field-enhanced QJ \\
\hline PCEo $(\%)$ & 5.48 & 6.17 \\
$\frac{\sigma}{\sqrt{N}}$ & 0.10 & 0.07 \\
\hline \hline
\end{tabular}

CQD layer was fabricated on an ITO-coated glass substrate using a dip-coating procedure that involved an ammonium hydroxide ligand exchange. This was followed by a layerby-layer spin-casting process for the n-type CQD film and solid state ligand exchange using tetrabutylammonium iodide in methanol, all carried out in an inert nitrogen atmosphere. The same procedure was followed to deposit the top N-layer where the size of quantum dots was varied. The top contacts consisted of $30 \mathrm{~nm}$ of sputtered aluminum-doped zinc oxide followed by $300 \mathrm{~nm}$ of thermally evaporated silver.

Figure 2 shows the experimental and, for comparison, also simulated current-voltage curves for the devices at AM1.5 illumination conditions. Measurements were performed in a nitrogen atmosphere using a solar spectrum simulator consisting of a Xenon lamp and filters. Illuminated device areas of $0.049 \mathrm{~cm}^{2}$ were defined using aperture masks. Current-voltage characteristics were measured using a Keithley 2400 source meter. The experimental device data follow similar trends as the simulated data. The points corresponding to the lowest electronic bandgap for the N-layer represent the Quantum Junction structure, used as a control. The best field-enhanced QJ device achieved a PCE of 6.6\%, a $12 \%$ increase over the control QJ efficiency of $5.9 \%$. Statistical analysis performed on 20 control and 20 FEQuantum Junction devices shows that the improvement in PCE is greater than the statistical error. The results are summarized in Table I. The increase in device efficiency was attributable to increases in both voltage and current in the optimized field-enhanced QJ device, as predicted by simulations (Fig. 3).

To explore further the potential to improve performance using the field-enhanced QJ structure, we explore using simulation the impact of different doping densities for the $\mathrm{N}$ layer. We present the results for a field-enhanced QJ employing the optimum bandgap for the $\mathrm{N}$ layer which maximizes the PCE as shown in Figure 2(d). The donor density within the N layer was varied widely across the range $10^{14}-10^{18} \mathrm{~cm}^{-3}$. The (a)

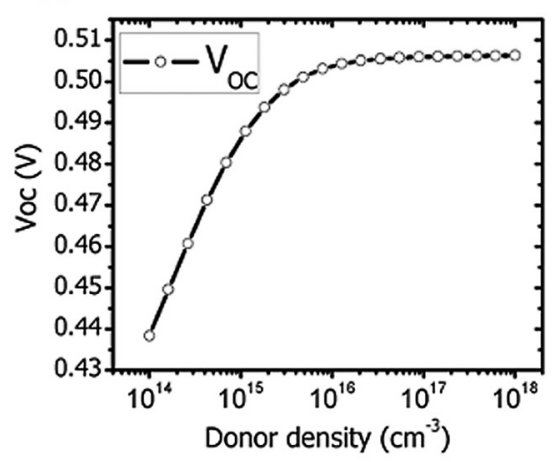

(b)

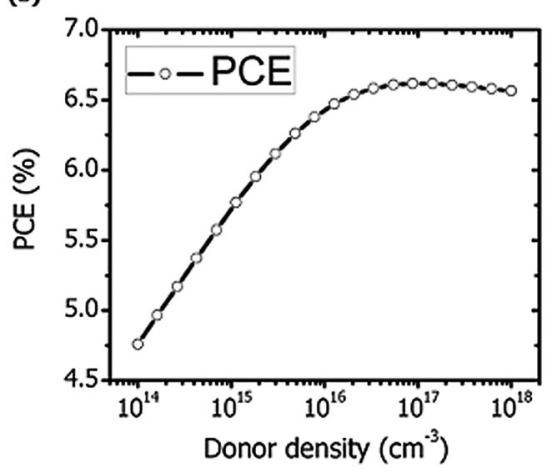

FIG. 4. Simulated $\mathrm{V}_{\mathrm{oc}}$ (a) and PCE (b) as a function of the donor density in the N layer for a field-enhanced QJ. 
doping of the $\mathrm{n}$ layer was kept fixed at $10^{16} \mathrm{~cm}^{-3}$, consistent with experimentally measured values (see supplementary material) and previously reported data. ${ }^{12}$ Figure 4 shows the simulated $\mathrm{V}_{\mathrm{OC}}$ and $\mathrm{PCE}$ as a function of the $\mathrm{N}$ layer donor density. As expected, the $\mathrm{V}_{\mathrm{OC}}$ increases proportionally to the donor density since a higher n-type doping increases the Fermi level splitting. The PCE experiences a similar trend but exhibits a maximum for very high donor density values, after which there is no significant enhancement. For such high doping concentration, the $\mathrm{N}$ layer is no longer fully depleted and a quasi-neutral region forms within the $\mathrm{N}$ layer, where carrier transport is less efficient. This reduces the $\mathrm{J}_{\mathrm{SC}}$ and PCE for very high doping levels in the $\mathrm{N}$ layer. Impact on other device parameters is shown in the supplementary material.

We have demonstrated that bandgap engineering in colloidal quantum dot solids can be used to tailor the electric field and produce improvements in photovoltaic device performance. We improved both open circuit voltage and short circuit current by adding a top CQD layer with an optimized larger bandgap to a QJ device. This concept can be extended to design improved architectures based on electric field tailoring for further efficiency improvements thanks to the facile quantum-size effect tuning possible in CQD solids.

The authors would like to acknowledge the technical assistance and scientific guidance of E. Palmiano, R. Wolowiec, and D. Kopilovic. This publication is based in part on work supported by Award KUS-11-009-21 made by King Abdullah University of Science and Technology (KAUST), by the Ontario Research Fund Research Excellence Program, and by the Natural Sciences and Engineering Research Council (NSERC) of Canada.

${ }^{1}$ L. Sun, J. J. Choi, D. Stachnik, A. C. Bartnik, B. R. Hyun, G. G. Malliaras, T. Hanrath, and F. W. Wise, Nat. Nanotechnol. 7, 369 (2012).

${ }^{2}$ G. Konstantatos, I. Howard, A. Fischer, S. Hoogland, J. Clifford, E. Klem, L. Levina, and E. H. Sargent, Nature 442, 180 (2006).

${ }^{3}$ G. Konstantatos, J. Clifford, L. Levina, and E. H. Sargent, "Sensitive solution-processed visible-wavelength photodetectors," Nat. Photonics 1, 531 (2007).
${ }^{4}$ T. P. Osedach, N. Zhao, T. L. Andrew, P. R. Brown, D. D. Wanger, D. B. Strasfeld, L.-Y. Chang, M. G. Bawendi, and V. Bulovic, ACS Nano 6, 3121 (2012).

${ }^{5}$ D. V. Talapin and C. B. Murray, Science 310, 86 (2005).

${ }^{6}$ A. G. Pattantyus-Abraham, I. J. Kramer, A. R. Barkhouse, X. Wang, G. Konstantatos, R. Debnath, L. Levina, I. Raabe, M. K. Nazeeruddin, M. Gratzel, and E. H. Sargent, ACS Nano 4, 3374 (2010).

${ }^{7}$ L. Etgar, W. Zhang, S. Gabriel, S. G. Hickey, M. K. Nazeeruddin, A. Eychmuller, B. Liu, and M. Gratzel, Adv. Mater. 24, 2202 (2012).

${ }^{8}$ Z. Ning, D. Zhitomirsky, V. Adinolfi, B. Sutherland, J. Xu, O. Voznyy, P. Maraghechi, X. Lan, S. Hoogland, Y. Ren, and E. H. Sargent, Adv. Mater. 25, 1719 (2013).

${ }^{9}$ R. J. Ellingson, M. C. Beard, J. C. Johnson, P. Yu, O. I. Micic, A. J. Nozik, A. Shabaev, and Alexander L. Efros, Nano Lett. 5, 865 (2005).

${ }^{10}$ D. A. R. Barkhouse, R. Debnath, I. J. Kramer, D. Zhitomirsky, A. G. Pattantyus-Abraham, L. Levina, L. Etgar, M. Grätzel, and E. H. Sargent, Adv. Mater. 23, 3134 (2011).

${ }^{11}$ H. Liu, J. Tang, I. J. Kramer, R. Debnath, G. I. Koleilat, X. Wang, A. Fisher, R. Li, L. Brzozowski, L. Levina, and E. H. Sargent, Adv. Mater. 23, 3832 (2011).

${ }^{12}$ J. Tang, H. Liu, D. Zhitomirsky, S. Hoogland, X. Wang, M. Furukawa, L. Levina, and E. H. Sargent, Nano Lett. 12, 4889 (2012).

${ }^{13}$ J. M. Luther, M. Law, M. C. Beard, Q. Song, M. O. Reese, R. J. Ellingson, and A. J. Nozik, Nano Lett. 8, 3488 (2008).

${ }^{14}$ N. Zhao, Tim P. Osedach, L. Y. Chang, S. M. Geyer, D. Wanger, M. T. Binda, A. C. Arango, M. G. Bawendi, and V. Bulovic, ACS Nano 4, 3743 (2010).

${ }^{15}$ B. R. Hyun, Y. W. Zhong, A. C. Bartnik, L. Sun, H. D. Abruna, F. W. Wise, J. D. Goodreau, J. R. Matthews, T. M. Leslie, and N. F. Borrelli, ACS Nano 2, 2206 (2008).

${ }^{16}$ See supplementary material at http://dx.doi.org/10.1063/1.4813074 for capacitance-voltage measurements, doping analysis simulations and simulated EQE.

${ }^{17}$ J. Nelson, The Physics of Solar Cells (Imperial College Press), p. 58.

${ }^{18}$ B. Qui and J. Wang, J. Mater. Chem. 22, 24315 (2012).

${ }^{19}$ D. Zhitomirsky, M. Furukawa, J. Tang, P. Stadler, S. Hoogland, O. Voznyy, H. Liu, and E. H. Sargent, Adv. Mater. 24, 6181 (2012).

${ }^{20}$ O. Voznyy, D. Zhitomirsky, P. Stadler, Z. Ning, S. Hoogland, and E. H. Sargent, ACS Nano 6, 8448 (2012).

${ }^{21}$ M. Burgelman, P. Nollet, and S. Degrave, Thin Solid Films 361, 527 (2000).

${ }^{22}$ D. Zhitomirsky, I. J. Kramer, A. J. Labelle, A. Fischer, R. Debnath, J. Pan, O. M. Bakr, and E. H. Sargent, Nano Lett. 12, 1007 (2012).

${ }^{23}$ I. J. Kramer and E. H. Sargent, ACS Nano 5, 8506 (2011).

${ }^{24}$ H. Liu, D. Zhitomirsky, S. Hoogland, J. Tang, I. J. Kramer, Z. Ning, and E. H. Sargent, Appl. Phys. Lett. 101, 151112 (2012).

${ }^{25}$ C. H. Henry, J. Appl. Phys. 51, 4494 (1980).

${ }^{26}$ M. Graetzel, René A. J. Janssen, David B. Mitzi, and Edward H. Sargent, Nature 488, 304-312 (2012). 